\title{
Off-label indications for antidepressants in primary care: descriptive study of prescriptions from an indication based electronic prescribing system
}

\author{
Jenna Wong, ${ }^{1}$ Aude Motulsky, ${ }^{1,2}$ Michal Abrahamowicz, ${ }^{1}$ Tewodros Eguale, ${ }^{3}$ David L Buckeridge, \\ Robyn Tamblyn ${ }^{1}$
}

${ }^{1}$ Department of Epidemiology, Biostatistics, and Occupational Health, McGill University, Montréal, Canada

${ }^{2}$ Centre de recherche du Centre hospitalier de l'Université de

Montréal, School of Public Health, University of Montréal,

Montréal, Canada

${ }^{3}$ Massachusetts College of Pharmacy and Health Sciences University, Boston, MA, USA

Correspondence to: J Wong jenna.wong@mail.mcgill.ca

Cite this as: $B M J$ J 2017;356:j603 http://dx.doi.org/10.1136/bmj.j603

Accepted: 18 January 2017

\section{ABSTRACT}

\section{OBJECTIVE}

To examine off-label indications for antidepressants in primary care and determine the level of scientific support for off-label prescribing.

\section{DESIGN}

Descriptive study of antidepressant prescriptions written by primary care physicians using an indication based electronic prescribing system.

SETTING

Primary care practices in and around two major urban centres in Quebec, Canada.

\section{PARTICIPANTS}

Patients aged 18 years or older who visited a study physician between 1 January 2003 and 30 September 2015 and were prescribed an antidepressant through the electronic prescribing system.

\section{MAIN OUTCOME MEASURES}

Prevalence of off-label indications for antidepressant prescriptions by class and by individual drug. Among off-label antidepressant prescriptions, the proportion of prescriptions in each of the following categories was measured: strong evidence supporting use of the prescribed drug for the respective indication; no strong evidence for the prescribed drug but strong evidence supporting use of another drug in the same class for the indication; or no strong evidence supporting use of the prescribed drug and all other drugs in the same class for the indication.

RESULTS

106850 antidepressant prescriptions were written by 174 physicians for 20920 adults. By class, tricyclic

\section{WHAT IS ALREADY KNOWN ON THIS TOPIC}

Off-label drug use without strong scientific evidence is associated with an increased risk of adverse drug events

About a third of all antidepressants in primary care are prescribed for off-label indications The degree to which off-label antidepressant prescriptions are supported by strong scientific evidence is unknown

\section{WHAT THIS STUDY ADDS}

Most off-label antidepressant prescriptions lack strong scientific evidence, but another evidence based antidepressant from the same class could often be considered as an alternative

There is an important need to produce more evidence evaluating the clinical outcomes associated with off-label antidepressant use

Indication based electronic prescribing systems represent an effective means to study off-label antidepressant use and communicate evidence back to physicians to optimise prescribing decisions

antidepressants had the highest prevalence of off-label indications (81.4\%, 95\% confidence interval, $77.3 \%$ to $85.5 \%$ ), largely due to a high off-label prescribing rate for amitriptyline $(93 \%, 89.6 \%$ to $95.7 \%$ ). Trazodone use for insomnia was the most common off-label use for antidepressants, accounting for $26.2 \%$ (21.9\% to $30.4 \%$ ) of all off-label prescriptions. For only $15.9 \%$ (13.0\% to $19.3 \%)$ of all off-label prescriptions, the prescribed drug had strong scientific evidence for the respective indication. For $39.6 \%$ (35.7\% to $43.2 \%$ ) of off-label prescriptions, the prescribed drug did not have strong evidence but another antidepressant in the same class had strong evidence for the respective indication. For the remaining $44.6 \%$ (40.2\% to $49.0 \%$ ) of off-label prescriptions, neither the prescribed drug nor any other drugs in the class had strong evidence for the indication.

\section{CONCLUSIONS}

When primary care physicians prescribed antidepressants for off-label indications, these indications were usually not supported by strong scientific evidence, yet often another antidepressant in the same class existed that had strong evidence for the respective indication. There is an important need to generate and provide physicians with evidence on off-label antidepressant use to optimise prescribing decisions.

\section{Introduction}

Antidepressant use has increased substantially in the $\mathrm{UK}^{12}$ and in other western countries such as Canada ${ }^{3}$ and the USA. ${ }^{4}$ In fact, the number of antidepressants dispensed in England increased by 3.9 million (6.8\%) between 2014 and 2015-more than any other therapeutic class of prescription drugs. ${ }^{2}$ One suspected factor underlying the widespread use of antidepressants is an expanding array of indications for these drugs, many of which are unapproved (off-label) for certain antidepressants. ${ }^{5}$

There is a lack of epidemiological evidence on the extent to which physicians prescribe antidepressants for off-label indications because treatment indications are not documented for most prescriptions. ${ }^{6}$ With the advent of electronic prescribing (e-prescribing) systems, however, formal documentation of treatment indications linked to prescriptions (that is, indication based prescribing) is possible. Although indication based prescribing is not broadly used at the moment, it represents a valuable means for studying off-label prescribing. ${ }^{7}$ We recently used data from a unique, indication based e-prescribing system to describe treatment 
indications for antidepressants in primary care. ${ }^{8}$ We found that over the past decade, primary care physicians commonly and increasingly prescribed antidepressants for non-depressive indications. Moreover, when antidepressants were not prescribed for depression, two of three prescriptions were for an off-label indication.

Off-label prescribing warrants particular attention and oversight when the drug use is not supported by scientific evidence showing greater benefits relative to risk. ${ }^{910}$ Inefficacious antidepressant use is a concern because it creates unnecessary costs and puts patients at risk of experiencing burdensome side effects and serious adverse events that could be avoided. For example, even though newer generation antidepressants such as selective serotonin reuptake inhibitors (SSRIs) are considered safer and more tolerable than the older generation tricyclic antidepressants (TCAs), they are costly and have still been associated with notable side effects and safety concerns. These side effects include sexual dysfunction, drowsiness, insomnia, weight gain, and fatigue, ${ }^{11-13}$ and safety concerns include an increased risk of fractures ${ }^{14}$ and upper gastrointestinal bleeds. ${ }^{1516}$ Off-label antidepressant use could also expose patients to unknown health risks if their clinical characteristics differ from the patient populations studied in pre-market clinical trials. ${ }^{17}$ Indeed, the risk of adverse drug events has been found to be $54 \%$ higher when drugs are used off-label without strong scientific evidence than when drugs are used on-label. ${ }^{18}$

Although an estimated $29 \%$ of antidepressants are prescribed for off-label indications, ${ }^{8}$ it is unknown to what extent these off-label prescriptions are supported by scientific evidence. Thus, the objective of this study was to examine off-label indications for antidepressants in primary care and assess the level of scientific evidence supporting these off-label prescriptions.

\section{Methods}

\section{Study design and setting}

This descriptive study took place in the Canadian province of Quebec, where a universal health insurance programme covers the cost of essential medical care for all residents. By law, all residents must be covered for prescription drugs through either private plans (that is, group or employee benefit plans) or the public drug insurance plan. About $50 \%$ of residents are registered in the public drug insurance plan, including those older than 65, welfare recipients, and those not insured through an employer. At a minimum, all private plans must provide the same formulary for insured drugs as the public drug insurance plan. ${ }^{19}$

\section{Data source and study population}

The Medical Office of the XXIst Century (MOXXI) is an electronic prescription and drug management system used by consenting primary care physicians in community based, fee-for-service practices around two major urban centres in Quebec. ${ }^{20}$ Since 2003, 207 physicians (25\% of eligible physicians) and over 100000 patients ( $26 \%$ of all who visited a MOXXI physician) have consented to participate in the MOXXI programme and have their information used for research purposes.

The e-prescribing tool in the MOXXI system requires physicians to explicitly record at least one treatment indication per prescription by either using a dropdown menu that lists on-label and off-label indications (without distinction) or typing the indication(s) into a free text field. In a validation study, ${ }^{21}$ these physician documented indications had excellent sensitivity (98.5\%) and high positive predictive value (97.0\%) when compared with a blinded, post hoc, physician facilitated chart review. The MOXXI system also provides physicians with access to professional drug monographs that are maintained by a commercial vendor ${ }^{22}$ and produces automated drug alerts about potential prescribing problems. Alerts are generated when potential dosing errors or drug-drug, drug-disease, drug-age, or drug-allergy contraindications are identified; however, alerts are not generated when drugs are prescribed for off-label indications. This study was approved by the McGill institutional review board.

\section{Inclusion and exclusion criteria}

This study included prescriptions of drugs approved for depression that were written by MOXXI physicians between 1 January 2003 and 30 September 2015 for patients aged 18 years or older. The antidepressant prescription was the unit of analysis. We excluded drugs with fewer than 150 prescriptions during the study period (roughly corresponding to a prescribing frequency of fewer than once per month). This resulted in the exclusion of all monoamine oxidase inhibitors (phenelzine, tranylcypromine, moclobemide, and isocarboxazid), nefazodone, maprotiline, and vortioxetine.

\section{Measurements}

On-label versus off-label indications

Treatment indications were first categorised by use of ICD-10 (international classification of diseases, 10th revision). Each prescription-representing a drug-indication pair-was then classified as on-label or off-label, depending on whether the drug had been approved for the indication by Health Canada or the US Food and Drug Administration as of September 2015 (the end of the study period). Approved indications were determined at the end of the study period rather than the year in which the prescription was written so that all prescriptions would be classified using the same benchmark. If a physician recorded multiple indications for the drug ( $n=1922,1.8 \%$ of all antidepressant prescriptions), the prescription was classified as off-label only if all the indications were not approved.

Level of scientific evidence for off-label prescriptions Off-label prescriptions were further analysed according to the level of scientific evidence supporting the drug's use for the off-label indication. Off-label prescriptions were assigned to one of three categories: strong evidence for the prescribed drug, no strong evidence for the prescribed drug but strong evidence for another drug in the same class, or no strong evidence for the 
prescribed drug and all other drugs in the same class. To determine whether off-label prescriptions had strong evidence for the prescribed drug, we used the DRUGDEX compendium (Thomson Micromedex), ${ }^{23}$ which is a reputable and authoritative reference used by the US Centers for Medicare and Medicaid Services to determine coverage for off-label drug uses. ${ }^{24}$ The compendium contains evaluations of drug efficacy, strength of recommendation, and strength of evidence for off-label drug indication pairs.

Using the same criteria as in previous studies, ${ }^{71825}$ we classified off-label prescriptions as having strong evidence for the prescribed drug if evidence showed that the drug was effective or favoured efficacy for the indication, the drug was recommended for all or most patients with the indication, and at least one randomised clinical trial was included among the studies used to evaluate the drug's efficacy for the indication. If an off-label prescription did not have strong evidence for the prescribed drug, we then determined whether there was strong evidence for another drug in the same class. This condition was satisfied if another drug in the same class was either on-label or off-label with strong evidence for the indication. If an off-label prescription still did not have strong evidence for another drug in the class, then the prescription was classified as having no strong evidence for the prescribed drug and all other drugs in the same class.

\section{Statistical analysis}

Patient and physician characteristics were summarised by use of descriptive statistics. The prevalence of off-label indications was estimated as the number of off-label prescriptions divided by the total number of antidepressant prescriptions overall, in the class, or for the individual drug. We estimated the level of scientific evidence for off-label prescriptions as the number of off-label prescriptions in each evidence category divided by the total number of off-label antidepressant prescriptions overall or in the class. The prevalence of different treatment indications for each drug was estimated as a proportion, using the total number of prescriptions for the drug as the denominator. For all proportions, we calculated 95\% confidence intervals using a cluster bootstrap approach ${ }^{26}$ to account for within-cluster correlation among prescriptions for the same patient and from the same physician. The reported 95\% confidence intervals correspond to the values of the 2.5th and 97.5th percentiles of the distribution of the respective estimates across 1000 bootstrap re-samples. ${ }^{26}$ All analyses were conducted using SAS (SAS Institute) software, version 9.4 .

\section{Patient involvement}

No patients were involved in setting the research question or the study measures, nor were they involved in developing plans for the design or implementation of the study. No patients were asked to advise on interpretation or writing up of results. The study findings will be disseminated to study participants through physician newsletters and patient-friendly handouts.

\section{Results}

During the study period, 106850 antidepressant prescriptions (5.8\% of 1.83 million prescriptions for any drug) were written by 174 primary care physicians for 20920 adults. There was roughly an equal number of male $(n=90 ; 52 \%)$ and female $(n=84 ; 48 \%)$ physicians, most of whom had been trained in North America $(n=160 ; 92 \%)$ and practicing for at least 15 years $(n=131$; $75 \%)$. Two thirds of patients were female $(n=13990$; $66.9 \%$ ), most patients were middle aged at the time of their earliest antidepressant prescription (median 53 years, interquartile range 43-65), and patients were equally likely to have public $(n=10875 ; 52.0 \%)$ or private $(n=10045 ; 48.0 \%)$ drug insurance. Over the study period, patients had a median of three (interquartile range 1-7) antidepressant prescriptions and were prescribed a median of one (1-2) type of antidepressant drug.

\section{Prevalence of off-label indications}

Overall, 29.3\% (95\% confidence interval $26.6 \%$ to $32.3 \%$ ) of all antidepressant prescriptions were written for an off-label indication (table 1). By class, TCAs had the highest prevalence of off-label indications $(81.4 \%$, $77.3 \%$ to $85.5 \%$ ), followed by other antidepressants (trazodone, bupropion, and mirtazapine; $42.4 \%, 37.1 \%$ to $47.7 \%$ ) and SSRIs (21.8\%, $19.0 \%$ to $25.0 \%)$. By contrast, the prevalence of off-label indications was much lower for serotonin-norepinephrine (noradrenaline) reuptake inhibitors (SNRIs; 6.1\%, 4.8\% to 7.5\%). The high prevalence of off-label indications for TCAs was mostly due to amitriptyline, which was only approved for depression but was almost exclusively prescribed for off-label indications $(93.0 \%, 89.6 \%$ to $95.7 \%)$-most commonly pain (48.4\%, 39.7\% to $57.8 \%)$, insomnia $(22.5 \%, 13.6 \%$ to $31.3 \%$ ), and migraine $(16.7 \%, 12.2 \%$ to $21.9 \%$; table 2$)$. The high prevalence of off-label indications among other antidepressants (trazodone, bupropion, and mirtazapine) was largely due to trazodone, which was mostly prescribed for insomnia (82.5\%, 74.5\% to 88.1\%) even though it was not approved for this indication. SSRIs and SNRIs had a lower prevalence of off-label indications because they were more frequently prescribed for depression than TCAs, which by definition was an approved indication for all antidepressants (table 2).

\section{Level of scientific evidence for off-label indications}

Among all off-label antidepressant prescriptions, there were 143 unique drug indication pairs-the most common of which were trazodone for insomnia (representing $26.2 \%$, $95 \%$ confidence interval $21.9 \%$ to $30.4 \%$, of all off-label prescriptions), citalopram for anxiety (17.8\%, $14.8 \%$ to $21.3 \%$ ), amitriptyline for pain $(13.8 \%, 11.0 \%$ to $16.9 \%)$, and amitriptyline for insomnia $(6.4 \%, 3.9 \%$ to $9.5 \%$; data not shown). Only three of these 143 off-label drug indication pairs met the predefined criteria ${ }^{71825}$ for having strong scientific evidence: amitriptyline (a TCA) for pain, escitalopram (an SSRI) for panic disorders, and venlafaxine (an SNRI) for obsessive compulsive disorder. 


\begin{tabular}{|c|c|c|c|c|c|c|c|c|}
\hline \multirow[b]{3}{*}{$\begin{array}{l}\text { Drug class } \\
\text { (No of prescriptions) }\end{array}$} & \multicolumn{2}{|c|}{ Off-label indication } & \multicolumn{6}{|c|}{ Level of evidence for off-label indications } \\
\hline & \multirow[b]{2}{*}{ No } & \multirow[b]{2}{*}{$\begin{array}{l}\text { Percentage* } \\
(95 \% \mathrm{CI})\end{array}$} & \multicolumn{2}{|c|}{$\begin{array}{l}\text { Strong evidence for } \\
\text { prescribed drug } \neq\end{array}$} & \multicolumn{2}{|c|}{$\begin{array}{l}\text { No strong evidence for prescribed } \\
\text { drug but strong evidence for } \\
\text { another drug in same class }\end{array}$} & \multicolumn{2}{|c|}{$\begin{array}{l}\text { No strong evidence for } \\
\text { prescribed drug and all other } \\
\text { drugs in same class }\end{array}$} \\
\hline & & & No & $\begin{array}{l}\text { Percentage§ } \\
(95 \% \mathrm{CIt})\end{array}$ & No & $\begin{array}{l}\text { Percentage§ } \\
(95 \% \mathrm{CIt})\end{array}$ & No & $\begin{array}{l}\text { Percentage§ } \\
(95 \% \mathrm{CI})\end{array}$ \\
\hline SSRI ( $\mathrm{n}=45608)$ & 9960 & 21.8 (19.0 to 25.0$)$ & 473 & 4.7 (2.7 to 7.2$)$ & 9160 & $92.0(89.2$ to 94.4$)$ & 327 & $3.3(2.0$ to 4.8$)$ \\
\hline SNRI $(n=25235)$ & 1539 & $6.1(4.8$ to 7.5$)$ & 169 & $11.0(4.6$ to 18.4$)$ & 544 & $35.4(25.0$ to 46.7$)$ & 826 & $53.7(40.6$ to 66.6$)$ \\
\hline TCA $(n=11645)$ & 9480 & $81.4(77.3$ to 85.5$)$ & 4335 & 45.7 (37.8 to 54.0$)$ & 2682 & 28.3 (20.5 to 36.6$)$ & 2463 & $26.0(21.2$ to 31.1$)$ \\
\hline Other $^{\star *}(n=24362)$ & 10340 & 42.4 (37.1 to 47.7$)$ & 0 & 0.0 (0.0 to 0.0$)$ & NA & NA & 10340 & $100.0(100.0$ to 100.0$)$ \\
\hline All classes $(n=106850)$ & 31319 & 29.3 (26.6 to 32.3$)$ & 4977 & 15.9 (13.0 to 19.3) & 12386 & 39.6 (35.7 to 43.2$)$ & 13956 & 44.6 (40.2 to 49.0) \\
\hline \multicolumn{9}{|c|}{$\begin{array}{l}\text { SSRI=selective serotonin reuptake inhibitors; SNRI=serotonin-norepinephrine reuptake inhibitors; TCA=tricyclic antidepressants; NA=not assessed for drugs in this category because they were } \\
\text { not considered as part of the same class. } \\
\text { *Calculated using the total number of prescriptions in the class as the denominator. } \\
\text { tCalculated by a cluster bootstrap approach } 26 \text { to account for non-independence of prescriptions from the same physician and for the same patient. Reported } 95 \% \text { confidence intervals } \\
\text { correspond to values at the } 2.5 \text { th and } 97.5 \text { th percentiles of the distribution of respective estimates across } 1000 \text { bootstrap re-samples. } \\
\text { fBased on evaluations from DRUGDEX compendium in three dimensions: efficacy, strength of recommendation, and strength of evidence. Prescriptions for an off-label indication were classified } \\
\text { as having strong evidence for a prescribed drug if evidence showed that the drug was effective or favoured efficacy for the indication, the drug was recommended for all or most patients with } \\
\text { the indication, and at least one randomised controlled trial was included among the studies used to evaluate the drug's efficacy for the indication. } \\
\S \text { Calculated using the number of prescriptions in the class that were written for an off-label indication as the denominator. } \\
\text { IOff-label prescriptions where the prescribed drug did not have strong evidence for the indication, but another drug in the class was either on-label or off-label with strong evidence for the } \\
\text { indication based on evaluations from the DRUGDEX compendium. }\end{array}$} \\
\hline
\end{tabular}

These three pairs collectively comprised $15.9 \%$ (13.0\% to 19.3\%) of all off-label antidepressant prescriptions (table 1) - most which were amitriptyline prescriptions for pain (representing $87.1 \%, 80.9 \%$ to $92.1 \%$, of all off-label prescriptions with strong evidence for the prescribed drug). As a result, the proportion of off-label antidepressant prescriptions with strong evidence for the prescribed drug was much higher for TCAs (45.7\%, 37.8\% to 54.0\%) than for SNRIs (11.0\%, $4.6 \%$ to $18.4 \%$ ) and SSRIs (4.7\%, $2.7 \%$ to $7.2 \%$; table 1 ).

Off-label antidepressant prescriptions had strong evidence for another drug in the same class-but not the prescribed drug-in 39.6\% (95\% confidence interval $35.7 \%$ to $43.2 \%$ ) of all cases (table 1). This proportion was highest among off-label SSRI prescriptions (92.0\%, $89.2 \%$ to $94.4 \%$ ), and lower among off-label prescriptions for SNRIs $(35.4 \%, 25.0 \%$ to $46.7 \%$ ) and TCAs $(28.3 \%, 20.5 \%$ to $36.6 \%)$. This proportion was not assessed for other antidepressants because trazodone, bupropion, and mirtazapine were not considered as part of the same class.

For the remaining $44.6 \%$ (95\% confidence interval $40.2 \%$ to $49.0 \%$ ) of off-label antidepressant prescriptions, neither the prescribed drug nor any other drug in the same class had strong evidence for the indication (table 1). All off-label prescriptions for other antidepressants (trazodone, bupropion, and mirtazapine) were classified in this evidence category. The proportion of off-label prescriptions with no scientific support for any drug in the class was also quite high for SNRIs (53.7\%, $40.6 \%$ to $66.6 \%$ ) and TCAs $(26.0 \%, 21.2 \%$ to $31.1 \%)$, but was much lower for SSRIs (3.3\%, 2.0\% to 4.8\%).

\section{Discussion}

This study provides evidence on the level of scientific support for off-label antidepressants prescriptions, the prevalence of off-label indications for individual antidepressants, and the most common off-label uses for antidepressants. Nearly a third (29\%) of all antidepressants in this study were prescribed for an off-label indication, as found previously. ${ }^{8}$ Among all off-label antidepressant prescriptions, only one in six prescriptions was supported by strong scientific evidence, but there was often another antidepressant in the same class with strong evidence that could have been considered instead, especially among off-label SSRI prescriptions. Still, nearly half of all off-label antidepressant prescriptions did not have strong evidence for the prescribed drug and all other antidepressants in the same class. Among the many off-label uses for antidepressants, physicians most frequently prescribed trazodone for insomnia even though this use was not evidence based.

\section{Comparison with other studies}

Few published studies exist on off-label prescribing, owing to challenges associated with measuring diagnoses (indications) for prescriptions. Compared with our findings where $29 \%$ of antidepressant prescriptions were off-label, Chen and colleagues ${ }^{27}$ found that $75 \%$ of people enrolled to Georgia Medicaid who were being treated with antidepressants received at least one antidepressant off-label. The rate of off-label antidepressant use was notably higher in this study because the authors classified prescriptions as off-label if the patient did not have a diagnostic code for an approved indication recorded in administrative claims data during the same year. This methodology most likely overestimated the off-label prescribing rate because diagnostic codes in administrative data are often incomplete or inaccurate, especially for psychiatric conditions. ${ }^{28}$

Only three studies-one Canadian ${ }^{7}$ and two US ${ }^{25} 29$ have used documented treatment indications to study off-label prescribing, none of which focused specifically on antidepressants. Eguale and colleagues ${ }^{7}$ combined antidepressants with other central nervous system drugs but reported fairly comparable results, with $26 \%$ of prescriptions for off-label indications-18\% of which 


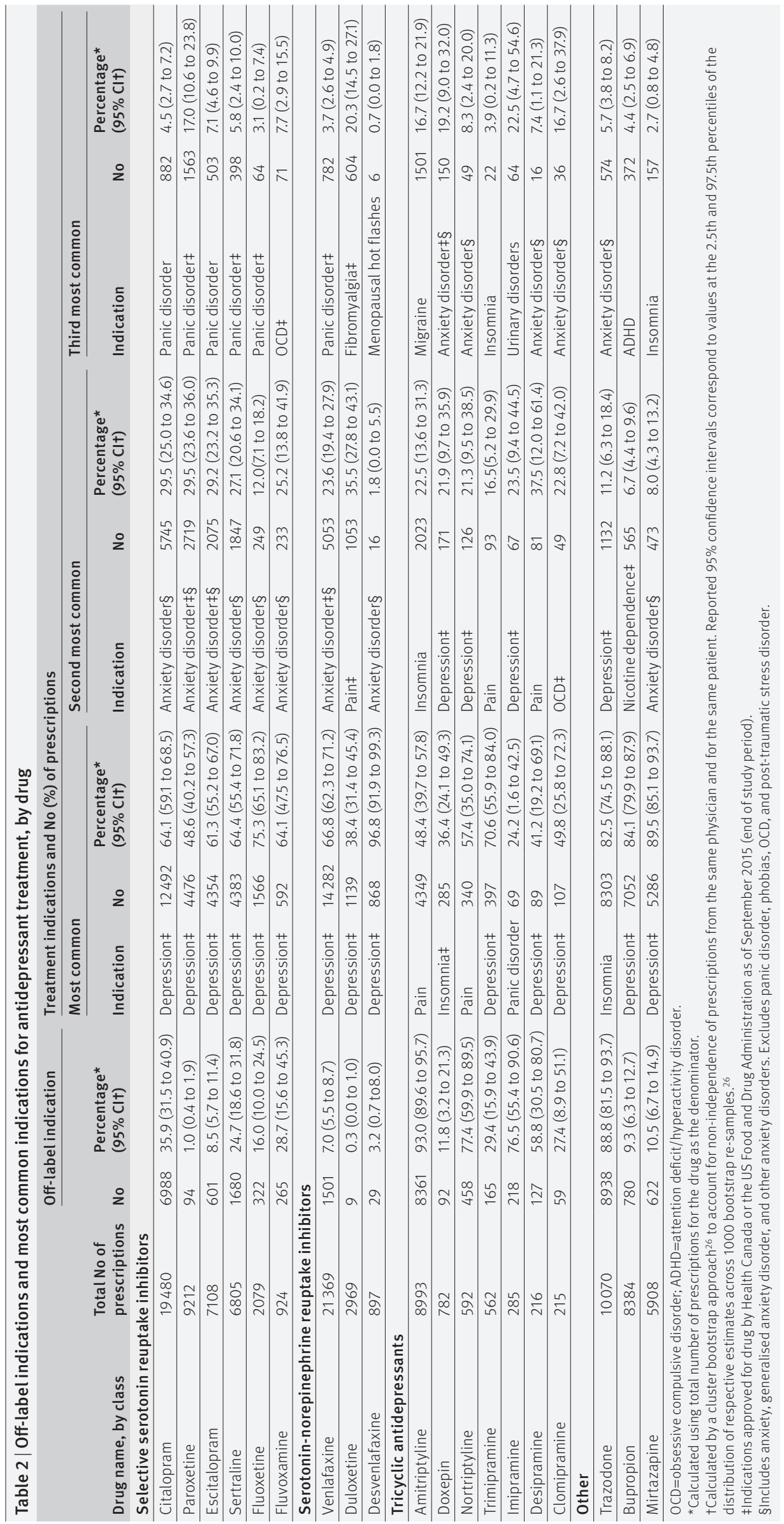


were supported by strong evidence. Radley and colleagues $^{29}$ combined antidepressants with anxiolytic and antipsychotic drugs, but again reported a similar off-label prescribing rate of 31\%. However, the proportion of off-label prescriptions with strong scientific support in this study was notably lower than ours at only $6 \%$, possibly due to the inclusion of other psychiatric drugs or because evidence to support some off-label antidepressant uses had not been generated at the time of the analysis. Finally, Walton and colleagues ${ }^{25}$ presented results for only five antidepressants but similarly found that amitriptyline and trazodone were the antidepressants most frequently prescribed for off-label indications. However, their off-label prescribing rate was notably lower for amitriptyline (69\%) and trazodone (43\%) than our rates, possibly reflecting inter-country differences in the use of antidepressants versus other drugs to treat pain and insomnia.

In all of these studies, none of the authors assessed the proportion of off-label antidepressant prescriptions where the prescribed drug did not have strong evidence but another antidepressant from the same class existed that had strong evidence for the respective indication.

\section{Potential explanations for off-label prescribing}

Several contextual factors could contribute to physicians prescribing antidepressants for off-label indications. Firstly, the vast and increasing number of drugs on the market makes it challenging for physicians to keep track of which indications are approved for specific products, ${ }^{30}$ especially when pharmaceutical companies have been known to promote drug use for off-label indications. ${ }^{31}$ Secondly, constraints such as the list of drugs included on patients' health plan formularies could influence which drugs physicians prescribe, especially if physicians presume that drugs in the same class are interchangeable. ${ }^{32} 33$ For example, in our setting, escitalopram was not covered for patients enrolled in the public drug insurance plan. We found that when study physicians prescribed SSRIs to patients with public drug insurance, they infrequently prescribed escitalopram (4.7\% of all SSRI prescriptions for patients with public drug insurance) but frequently prescribed citalopram (51.4\%). However, for patients with private drug insurance, study physicians equally prescribed escitalopram and citalopram (29.3\% and 31.7\% of all SSRI prescriptions for patients with private drug insurance, respectively).

Thirdly, primary care physicians might prescribe antidepressants off-label because alternative treatments for a given indication are contraindicated or perceived as higher risk medications. For example, benzodiazepines and $\mathrm{Z}$ drugs such as zolpidem and zaleplon have been shown to be efficacious for treating insomnia. ${ }^{34}$ However, these drugs have been labelled as potentially inappropriate treatments for older adults, and if prescribed, could even negatively affect providers' quality and performance measures. ${ }^{35}$ Many physicians who are concerned about the health of their older patients might consequently prescribe trazodone instead because they believe it is a safer treatment.
Finally, many off-label indications for antidepressants are symptom based conditions for which few approved drug treatments exist. Primary care physicians could be struggling to find effective treatments for these conditions and thus prescribe antidepressants as a last resort, indicating a gap in needed pharmacotherapy.

\section{Implications of findings}

For both primary care physicians and specialists (since specialists could initiate antidepressant treatment that is then continued by a primary care physician), our findings emphasise the importance of considering the level of evidence supporting risk-benefit when prescribing an antidepressant, especially if the drug is known to have important adverse side effects. ${ }^{36}$ When evidence to support efficacy is lacking, physicians should exercise caution, prescribe conservatively, and inform patients of this information via a shared decision making process. $^{36}$ This ideal, however, is challenging to achieve because physicians face time constraints, the drug market and scientific literature are vast and ever-evolving, and many physicians find it challenging to critically appraise and interpret the results of epidemiological studies. ${ }^{37}$ Indication based e-prescribing systems that are integrated with clinical decision support tools could help overcome these obstacles by notifying physicians when drugs are being prescribed off-label without supporting evidence and providing them with access to concise, up-to-date summaries of the available evidence. Providing the public with access to patient friendly resources about the level of scientific evidence supporting different treatment options for a given indication could further facilitate the decision making process between physicians and patients.

Our finding that among off-label prescriptions, 40\% were for indications where the prescribed drug did not have strong evidence but another drug in the same class was approved or supported by strong evidence is clinically important. Many physicians might view this type of off-label prescribing as different from off-label prescribing without scientific evidence for the entire class because they assume that drugs within the same class are interchangeable. ${ }^{38} 39$ However, class effects cannot be assumed because even slight differences in chemical structure between drugs can alter their pharmacodynamic and pharmacokinetic properties, leading to clinically relevant differences in efficacy and risk. ${ }^{39}$ For example, statins have been shown to differ not only in efficacy ${ }^{40}$ but also in safety, as demonstrated by the withdrawal of cerivastatin from the market in 2001 because the risk of rhabdomyolysis was 10 times higher for cerivastatin than other statins. ${ }^{41}$ Clinical guidelines recommend that when physicians select a particular drug to prescribe, they should consider the level of scientific evidence supporting the specific drug. ${ }^{42}$ It should not be assumed that all drugs within a class are likely to be efficacious for treating an indication when one member of the class has proven efficacy.

Finally, more evidence is needed on the clinical outcomes associated with antidepressant use for off-label indications. However, within a context of limited 
resources, it is unlikely that randomised clinical trials will be conducted for each off-label drug-indication pair, especially for older drugs that are no longer owned by an innovator company. ${ }^{9}$ Thus, in addition to randomised clinical trials, post-market drug surveillance systems represent valuable resources for assessing off-label antidepressant use. Such systems face challenges associated with measuring treatment indications and patient reported outcomes, but these challenges could be overcome by increasing the use of indication based e-prescribing systems and electronic health records that track patient outcomes. Indeed, this study demonstrates the benefits that indication based prescribing can have towards addressing knowledge gaps around off-label antidepressant prescribing.

\section{Strengths and limitations}

The key strength of this study is that it included more than 12 years of antidepressant prescriptions from an e-prescribing system where physicians systematically documented treatment indications at the point of prescribing. However, study participants were from one Canadian province where prescribers were generally younger and patients were generally older with more health complexities. ${ }^{43}$ These characteristics could influence the generalisability of our findings, because younger physicians are more likely to prescribe drugs off-label without scientific evidence, and patients with more health complexities are less likely to receive off-label prescriptions. ${ }^{7}$

Another study strength is that physicians were unlikely to have altered their true responses when recording indications in the e-prescribing system because the dropdown menu did not distinguish between on-label and off-label indications for a drug. On the other hand, we could not identify when physicians consciously prescribed antidepressants off-label. Indeed, a portion of antidepressants in this study might have been prescribed off-label for a specific reason (eg, patient experienced side effects to another drug in the same class, or formulary restrictions).

\section{Study considerations}

Firstly, our estimates of off-label antidepressant prescribing were conservative because we did not consider other aspects of off-label drug use (eg, dose, frequency, duration of treatment), and we used the approved indications and available evidence at the end of the study period. Secondly, we presumed that approved indications for drugs were backed by strong scientific evidence, which might not have been true in some cases given that the quality of clinical trial evidence used by regulatory agencies as the basis for approving new therapeutics and supplemental indications has been shown to vary widely. 4445

Thirdly, to identify evidence based off-label uses for antidepressants, we used pre-established criteria that has been used in other studies. ${ }^{71825}$ However, our list of evidence based antidepressants for each indication might not always be identical to the recommendations from clinical guidelines. For example, recommendations from two national guidelines for managing anxiety related disorders ${ }^{4246}$ are similar but slightly more inclusive than ours. Finally, because regulatory bodies in North America and Europe are not entirely harmonised in their list of approved indications for drugs, slight discrepancies in the rate of off-label antidepressant use could exist between North America and Europe.

\section{Conclusions}

By using information from an indication based e-prescribing system, we found that when primary care physicians prescribed antidepressants for off-label indications, the prescribed drug was usually not supported by strong evidence for the respective indication. However, there was often another drug in the same class with strong evidence that could have been considered. These findings highlight an urgent need to produce more evidence on the risks and benefits of off-label antidepressant use and to provide physicians with this evidence at the point of prescribing. Technologies such as indication based e-prescribing systems and electronic health records have the potential to become essential components of effective post-market drug surveillance systems for monitoring and evaluating off-label antidepressant use. By integrating these technologies with knowledge databases and clinical decision support tools, they could also provide an effective means for communicating evidence back to physicians to optimise prescribing decisions.

We thank Claude Dagenais (academic adviser, Faculty of Pharmacy, University of Montréal) for reviewing the manuscript and providing substantive comments.

Contributors: JW extracted and had full access to all of the study data and takes responsibility for the integrity of the data and the accuracy of the data analysis. JW contributed to the study design; analysis and interpretation of the data; drafting of the manuscript; and critical revision of the manuscript for important intellectual content. AM and RT contributed to the study design; analysis and interpretation of the data; and critical revision of the manuscript for important intellectual content. MA and TE contributed to the analysis and interpretation of the data; and critical revision of the manuscript for important intellectual content. DB contributed to the interpretation of the data and critical revision of the manuscript for important intellectual content. All authors read and approved the final manuscript. JW is the guarantor.

Funding: This study was funded by the Canadian Institutes of Health Research (CIHR; grant IOP-112675). JW is supported by the Vanier Canada Graduate Scholarship (CIHR) and the Max E Binz Fellowship (Faculty of Medicine, McGill University). MA is a James McGill professor of biostatistics at McGill University. The funders had no role in the study design; collection, analysis, interpretation of the data; writing of the manuscript; or in the decision to submit the manuscript for publication. Competing interests: All authors have completed the ICMJE uniform disclosure form at www.icmje.org/coi_disclosure.pdf and declare: support from the Canadian Institutes of Health Research for the submitted work; no financial relationships with any organisations that might have an interest in the submitted work in the previous three years; no other relationships or activities that could appear to have influenced the submitted work.

Ethical approval: This study was approved by the McGill institutional review board.

Data sharing: No additional data available.

The lead author affirms that the manuscript is an honest, accurate, and transparent account of the study being reported, and that no important aspects of the study have been omitted; and that any discrepancies from the study as planned (and, if relevant, registered) have been explained.

This is an Open Access article distributed in accordance with the Creative Commons Attribution Non Commercial (CC BY-NC 4.0) license, which permits others to distribute, remix, adapt, build upon this work 
non-commercially, and license their derivative works on different terms, provided the original work is properly cited and the use is non-commercial. See: http://creativecommons.org/licenses/ by-nc/4.0/

1 Moore M, Yuen HM, Dunn N, Mullee MA, Maskell J, Kendrick T. Explaining the rise in antidepressant prescribing: a descriptive study using the general practice research database. BMJ 2009;339:b3999. doi:10.1136/bmj.b3999.

2 Prescribing and Medicines Team, Health and Social Care Information Centre. Prescriptions dispensed in the community: England 2005-2015. 2016. https://digital.nhs.uk/catalogue/PUB20664/ pres-disp-com-eng-2005-15-rep.pdf

3 Hemels MEH, Koren G, Einarson TR. Increased use of antidepressants in Canada: 1981-2000. Ann Pharmacother 2002;36:1375-9. doi:10.1345/aph.1A331.

4 National Center for Health Statistics. Health, United States, 2010 with special feature on death and dying. 2011. https://www.cdc.gov/nchs/ data/hus/hus10.pdf

5 Stone KJ, Viera AJ, Parman CL. Off-label applications for SSRIs. Am Fam Physician 2003:68:498-504.

6 Li Y, Salmasian H, Harpaz R, Chase H, Friedman C. Determining the reasons for medication prescriptions in the EHR using knowledge and natural language processing. AMIA Annu Symp Proc 2011;2011:768-76

7 Eguale T, Buckeridge DL, Winslade NE, Benedetti A, Hanley JA, Tamblyn R. Drug, patient, and physician characteristics associated with off-label prescribing in primary care. Arch Intern Med 2012;172:781-8. doi:10.1001/archinternmed.2012.340.

8 Wong J, Motulsky A, Eguale T, Buckeridge DL, Abrahamowicz M, Tamblyn R. Treatment indications for antidepressants prescribed in primary care in Quebec, Canada, 2006-2015. JAMA 2016;315:2230-2. doi:10.1001/jama.2016.3445.

9 Dresser R, Frader J. Off-label prescribing: a call for heightened professional and government oversight. J Law Med Ethics 2009;37:476-86, 396. doi:10.1111/j.1748-720X.2009.00408.X

10 O'Malley PG. What does off-label prescribing really mean?Arch Intern Med 2012;172:759-60.

$11 \mathrm{HuXH}$, Bull SA, Hunkeler EM, et al. Incidence and duration of side effects and those rated as bothersome with selective serotonin reuptake inhibitor treatment for depression: patient report versus physician estimate. J Clin Psychiatry 2004;65:959-65. doi:10.4088/ JCP.v65n0712.

12 Cascade E, Kalali AH, Kennedy SH. Real-world data on SSR antidepressant side effects. Psychiatry (Edgmont) 2009;6:16-8.

13 Carvalho AF, Sharma MS, Brunoni AR, Vieta E, Fava GA. The safety, tolerability and risks associated with the use of newer generation antidepressant drugs: a critical review of the literature. Psychother Psychosom 2016;85:270-88. doi:10.1159/000447034.

14 Eom C-S, Lee H-K, Ye S, Park SM, Cho K-H. Use of selective serotonin reuptake inhibitors and risk of fracture: a systematic review and meta-analysis. J Bone Miner Res 2012;27:1186-95. doi:10.1002/ jbmr.1554.

15 Anglin R, Yuan Y, Moayyedi P, Tse F, Armstrong D, Leontiadis GI. Risk of upper gastrointestinal bleeding with selective serotonin reuptake inhibitors with or without concurrent nonsteroidal anti-inflammatory use: a systematic review and meta-analysis. Am J Gastroenterol 2014:109:811-9 doi:10.1038/ajg.2014.82.

16 Dall M, Schaffalitzky de Muckadell OB, Lassen AT, Hansen JM, Hallas J. An association between selective serotonin reuptake inhibitor use and serious upper gastrointestinal bleeding. Clin Gastroenterol Hepatol 2009·7:1314-21. doi:10.1016/i.cgh.2009.08.019.

17 Wittich CM, Burkle CM, Lanier WL. Ten common questions (and their answers) about off-label drug use. Mayo Clin Proc 2012;87:982-90. doi:10.1016/j.mayocp.2012.04.017.

18 Eguale T, Buckeridge DL, Verma A, et al. Association of off-label drug use and adverse drug events in an adult population. JAMA Intern Med 2016;176:55-63. doi:10.1001/jamainternmed.2015.6058.

19 Pomey M-P, Forest P-G, Palley HA, Martin E. Public/private partnerships for prescription drug coverage: policy formulation and outcomes in Quebec's universal drug insurance program, with comparisons to the Medicare prescription drug program in the United States. Milbank Q 2007;85:469-98. doi:10.1111/j.1468-0009.2007.00496.x

20 Tamblyn R, Huang A, KawasumiY, et al. The development and evaluation of an integrated electronic prescribing and drug management system for primary care. J Am Med Inform Assoc 2006;13:148-59. doi:10.1197/jamia.M1887.

21 Eguale T, Winslade N, Hanley JA, Buckeridge DL, Tamblyn R. Enhancing pharmacosurveillance with systematic collection of treatment indication in electronic prescribing: a validation study in Canada. Drug Saf 2010;33:559-67. doi:10.2165/11534580-000000000-00000

22 Vigilance Santé. www.vigilance.ca/en/

23 Thomson Micromedex. Drugdex system (internet database) Greenwood Village, Colo. https://micromedex.com/compendia
24 Center for Medicare Advocacy. CMA report: Medicare coverage for off-label drug use. 2010. www.medicareadvocacy.org/ cma-report-medicare-coverage-for-off-label-drug-use/

25 Walton SM, Schumock GT, Lee K-V, Alexander GC, Meltzer D, Stafford RS. Prioritizing future research on off-label prescribing: results of a quantitative evaluation. Pharmacotherapy 2008;28:1443-52. doi:10.1592/phco.28.12.1443.

26 Xiao Y, Abrahamowicz M. Bootstrap-based methods for estimating standard errors in Cox's regression analyses of clustered event times. Stat Med 2010:29:915-23 doi:10.1002/sim.3807.

27 Chen H, Reeves JH, Fincham JE, Kennedy WK, Dorfman JH, Martin BC. Off-label use of antidepressant, anticonvulsant, and antipsychotic medications among Georgia medicaid enrollees in 2001. / Clin Psychiatry 2006;67:972-82. doi:10.4088/JCP.v67n0615.

28 Rost K, Smith R, Matthews DB, Guise B. The deliberate misdiagnosis of major depression in primary care. Arch Fam Med 1994;3:333-7. doi:10.1001/archfami.3.4.333.

29 Radley DC, Finkelstein SN, Stafford RS. Off-label prescribing among office-based physicians. Arch Intern Med 2006;166:1021-6. doi:10.1001/archinte.166.9.1021.

30 Chen DT, Wynia MK, Moloney RM, Alexander GCUS. U.S. physician knowledge of the FDA-approved indications and evidence base for commonly prescribed drugs: results of a national survey. Pharmacoepidemiol Drug Saf 2009:18:1094-100. doi:10.1002/ pds.1825.

31 Ghinea N, Lipworth W, Kerridge I. Off-label promotion of prescription medicine is it ever justifiable?Ther Innov Regul Sci 2015;49:359-63doi:10.1177/2168479015570337.

32 Sbarbaro JA. Can we influence prescribing patterns? Clin Infect Dis 2001;33(Suppl 3):S240-4. doi:10.1086/321856

33 Stafford RS. Regulating off-label drug use--rethinking the role of the FDA. N Engl J Med 2008;358:1427-9. doi:10.1056/NEJMp0802107.

34 Wilson SJ, Nutt DJ, Alford C, et al. British Association for Psychopharmacology consensus statement on evidence-based treatment of insomnia, parasomnias and circadian rhythm disorders. J Psychopharmacol 2010;24:1577-601 doi:10.1177/0269881110379307.

35 Campanelli CM. American Geriatrics Society 2012 Beers Criteria Update Expert Panel. American Geriatrics Society updated Beers Criteria for potentially inappropriate medication use in older adults. J Am Geriatr Soc 2012:60:616-31. doi:10.1111/j.1532-5415.2012.03923.x

36 Largent EA, Miller FG, Pearson SD. Going off-label without venturing off-course: evidence and ethical off-label prescribing. Arch Intern Med 2009;169:1745-7. doi:10.1001/archinternmed.2009.314.

37 Godwin M, Seguin R. Critical appraisal skills of family physicians in Ontario, Canada. BMC Med Educ 2003;3:10. doi:101186/1472-6920-3-10

38 McAlister FA, Laupacis A, Wells GA, Sackett DL. Evidence-Based Medicine Working Group. Users' Guides to the Medical Literature: XIX Applying clinical trial results B. Guidelines for determining whether a drug is exerting (more than) a class effect. JAMA 1999;282:1371-7. doi:10.1001/jama.282.14.1371.

39 Johnston A, Stafylas P, Stergiou GS. Effectiveness, safety and cost of drug substitution in hypertension. Br I Clin Pharmacol 2010:70:32034. doi:10.1111/j.1365-2125.2010.03681.x

40 Dieleman JP, van Wyk JT, van Wijk MAM, et al. Differences between statins on clinical endpoints: a population-based cohort study. Curr Med Res Opin 2005;21:1461-8. doi:10.1185/030079905X61866.

41 Furberg CD, Pitt B. Withdrawal of cerivastatin from the world market. Curr Control Trials Cardiovasc Med 2001;2:205-7. doi:10.1186/ CVM-2-5-205.

42 Baldwin DS, Anderson IM, Nutt DJ, et al. Evidence-based pharmacological treatment of anxiety disorders, post-traumatic stress disorder and obsessive-compulsive disorder: a revision of the 2005 guidelines from the British Association for Psychopharmacology. J Psychopharmacol 2014;28:403-39. doi:10.1177/0269881114525674.

43 Bartlett G, Tamblyn R, Kawasumi Y, Poissant L, Taylor L. Nonparticipation bias in health services research using data from an integrated electronic prescribing project: The role of informed consent. Acta Bioeth 2005;11:145-59doi:10.4067/ S1726-569X2005000200005.

44 Downing NS, Aminawung JA, Shah ND, Krumholz HM, Ross JS. Clinical trial evidence supporting FDA approval of novel therapeutic agents, 2005-2012. JAMA 2014;311:368-77. doi:10.1001/jama.2013.282034.

45 Wang B, Kesselheim AS. Characteristics of efficacy evidence supporting approval of supplemental indications for prescription drugs in United States, 2005-14: systematic review. BM 2015:351:h4679 doi:10.1136/bmi.h4679.

46 Katzman MA, Bleau P, Blier P, et al. Canadian Anxiety Guidelines Initiative Group on behalf of the Anxiety Disorders Association of Canada/Association Canadienne des troubles anxieux and McGill University. Canadian clinical practice guidelines for the management of anxiety, posttraumatic stress and obsessive-compulsive disorders. BMC Psychiatry 2014;14(Suppl 1):S1. doi:10.1186/1471-244X-14-S1-S1. 